\title{
Enhancement of Antibody-Induced Arthritis via Toll-Like Receptor 2 Stimulation Is Regulated by Granulocyte Reactive Oxygen Species
}

Tiina Kelkka, ${ }^{* \dagger \ddagger}$ Malin Hultqvist, ${ }^{\S}$ Kutty Selva Nandakumar, ${ }^{\dagger}$ and Rikard Holmdahl ${ }^{\star \dagger}$

From the Medicity Research Laboratory," Turku, Finland; Medical Inflammation Research, ${ }^{\dagger}$ Department of Medical Biochemistry and Biophysics, Karolinska Institutet, Stockholm, Sweden; the Turku Doctoral Programme of Biomedical Sciences, ${ }^{\ddagger}$ Turku, Finland; and Medical Inflammation Research, ${ }^{\circledR}$ Lund University, Lund, Sweden

The suppressive role of phagocyte nicotinamide adenine dinucleotide phosphate (NADPH) oxidase (NOX2) complex-derived reactive oxygen species (ROS) in adaptive immunity-driven arthritis models is well established. In this study, we aimed to investigate the role of NOX2 complex-derived ROS in a model of innate immunity-driven arthritis and to identify the ROS-regulated innate receptors that control arthritis. We used collagen antibody-induced arthritis (CAIA), which is a T and B lymphocyte-independent model of the effector phase of arthritis and is induced by well-defined monoclonal arthritogenic antibodies and enhanced by injection of lipopolysaccharide (LPS). CAIA was induced in both wild-type and Ncf1 mutant mice that lack phagocyte oxidative burst, and stimulated with LPS and other agents to activate innate immune responses. We found that both LPS and lipomannan enhanced CAIA more potently in the presence of functional phagocyte ROS production than in its absence. The ROS-dependent enhancement of CAIA was regulated by TLR2, but not by TLR4 stimulation, and was driven by granulocytes, whereas macrophages did not contribute to the phenotype. In addition, we report that collagen-induced arthritis was not affected by the functionality of the TLR4. We report that TLR2 signaling as an important ROS-regulated proinflammatory pathway leads to severe neutrophil-dependent inflammation in murine CAIA and conclude that the TLR2 pathway is modulated by phagocyte ROS to stimulate the development of arthritis. (Am J Pathol 2012, 181 : 141-150; bttp://dx.doi.org/10.1016/j.ajpath.2012.03.031)

T cell-mediated arthritis models such as collagen-induced arthritis $(\mathrm{ClA})$ and pristane-induced arthritis share many characteristics with the human disease rheumatoid arthritis (RA). However, these experimental models have limitations when studying the acute inflammatory processes taking place in the inflamed joints. In T cell-dependent arthritis models, the clinically active disease is preceded by a priming phase, and this early phase is differently regulated than the following erosive local inflammation in the synovial tissue. ${ }^{1}$ To separate the adaptive priming response and the innate destructive acute joint inflammation, we and others ${ }^{2,3}$ have developed arthritis models that are independent of $\mathrm{T}$ lymphocyte function and are induced by collagen type II (CII) autoantibodies resulting in acute local joint inflammation in experimental animals.

Collagen antibody-induced arthritis (CAIA) is a T and B lymphocyte-independent model of the effector phase of arthritis. ${ }^{4}$ In most mouse strains, including the C57BI/ 10.Q-rhd (B10.Q) mice used in this work, antibody injection alone induces mild arthritis, and thus, for most experimental needs, joint inflammation is enhanced by an additional immunostimulatory agent. Lipopolysaccharide (LPS) is the routinely used arthritis enhancer ${ }^{2}$; lipomannan (LM) and Pam-3-cys, both TLR2 agonists, have also been shown to efficiently enhance and prolong antibodyinduced joint inflammation. 5,6 The use of immunological stimulants not only enhances joint inflammation, but also creates an opportunity to study the molecular mechanisms involved in arthritis induction and regulation in a controlled experimental set-up.

Many innate receptors and their ligands have been associated with arthritis initiation, enhancement, or suppression. There are reports linking most Toll-like receptors with arthritis regulation, ${ }^{7-16}$ and similarly, many other innate stimulants and receptors, such as peptidoglycan, ${ }^{17}$ zymosan, ${ }^{18}$ dectin-1, and NOD2, ${ }^{19,20}$ are also known to modulate joint inflammation. However, current literature is not consistent, and innate recep-

Supported by the Sigrid Juselius Foundation, Academy of Finland, Nordic Center of Excellence in Disease Genetics, Maire Lisko Foundation, the Swedish Science Strategic Foundation (SSF), and the EU project Masterswitch (HEALTH-F2-2008-223404).

Accepted for publication March 12, 2012.

Current address of M.H., Redoxis AB, Lund, Sweden.

Address reprint requests to Rikard Holmdahl, M.D., Ph.D., Medicity, University of Turku, Tykistökatu 6 A, FIN-20520 Turku, Finland. E-mail: Rikard.Holmdahl@ki.se. 
tors such as TLR2, TLR3, and TLR7 are not only reported to enhance ${ }^{5,6,9,13}$ arthritis, but also to suppress ${ }^{7,10,14}$ inflammatory signaling. The ultimate effect of any ligand/receptor depends on many factors, including the rodent strain, arthritis model, combinatorial effects of other stimulants present in the system, and environmental factors such as the animal house microbiota.

Phagocyte NADPH oxidase (NOX2) complex-derived reactive oxygen species (ROS) are known to suppress $T$ cell-mediated models of autoimmune arthritis in both rats and mice, ${ }^{21-23}$ but so far, their role during the effector phase of arthritis is largely unknown.

In this work, we studied how NOX2 complex-derived ROS affect CAIA, a well-established antibody-induced arthritis model.

\section{Materials and Methods}

\section{Animals}

All mice were housed in specific pathogen-free conditions in a controlled environment with standard bedding and controlled light cycles, and had access to standard food pellets and drinking water ad libitum. All experimental procedures were performed according to European Union standards, and the ethical permits were approved by the local authorities in Turku, Finland (ESHL-200802873/ym-23 and ESAVI-497/041003/2011) and Lund, Sweden (M-107-07 and M109-07).

All mouse strains were backcrossed onto the C57BL/ 10.Q.rhd genetic background, a strain with a DBA $/ 1 H-2^{9}$ fragment on a C57BL/10 background maintained by sisterbrother mating in our animal house, referred to in short as B10.Q. ${ }^{24}$ The point mutated Ncf1 gene (the coded protein also known as p47phox) was backcrossed onto the B10.Q background, and the B10.Q.Ncf1 ${ }^{\mathrm{m1} / \mathrm{J} / \mathrm{j}}$ (abbreviated $\mathrm{Ncfl}^{* / *}$ ) mice were ascertained to be genetically clean even in the linked fragment. ${ }^{22}$ The transgenic B10.Q. Ncf1 ${ }^{\mathrm{m} 1 \mathrm{~J} / \mathrm{m} 1 \mathrm{j}_{-}}$ $\mathrm{Tg}\left(\mathrm{Ncf1}\right.$ )1Rhd (in short, $\mathrm{MN}^{+}$) strain carried a transgene expressing functional Ncf1 predominantly on macrophages using the human CD68 promoter. ${ }^{25}$ The transgene was always bred heterozygous, and mice denoted as $\mathrm{MN}^{-}$are $\mathrm{Ncf1}^{* / *}$ littermate controls.

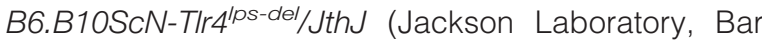
Harbor, ME) mice are defective in their LPS response and have a spontaneous mutation impairing TLR4 mRNA and protein expression. ${ }^{5,26,27}$ This mutation was crossed onto the B10.Q background (named in short as TLR4-def) and genotyped as described in Thomas et al. ${ }^{28}$ Mice were age and sex matched, and evaluated blindly for arthritis development in all experiments.

\section{Collagen Antibody-Induced Arthritis}

Cll-specific B-cell hybridomas were generated and characterized previously. ${ }^{29-31}$ All of the antibody-secreting clones were cultured in CL-1000 flasks (Integra Biosciences, Walliselen, Switzerland) using Dulbecco's Glutamax-I medium containing ultra-low bovine IgG (Gibco BRL, Invitrogen, Stockholm, Sweden). Antibodies from clone M2139 (IgG2b) bind J1 epitope of CII (551-564; GERGAAGIAGPK), CIIC1
Table 1. Immunostimulants Used to Stimulate CAIA

\begin{tabular}{|c|c|c|c|}
\hline Substance & Dose & Main receptors & $\begin{array}{l}\text { Effect on } \\
\text { CAIA }\end{array}$ \\
\hline LPS & $25 \mu \mathrm{g}$ & TLR4 and TLR2 & $\begin{array}{l}\text { Strong } \\
\text { enhancer }\end{array}$ \\
\hline LM & $35 \mu \mathrm{g}$ & TLR2 & $\begin{array}{l}\text { Strong } \\
\text { enhancer }\end{array}$ \\
\hline MPL & $25 \mu \mathrm{g}$ & TLR4 & Enhancer \\
\hline Peptidoglycan & $200 \mu \mathrm{g}$ & $\begin{array}{l}\text { TLR2, NOD1, } \\
\text { NOD2, etc. }\end{array}$ & No effect \\
\hline Pertussis toxin & $400 \mathrm{ng}$ & Multiple targets & No effect \\
\hline Flagellin & $7 \mu \mathrm{g}$ & TLR5 & No effect \\
\hline Zymosan & $2 \mathrm{mg}$ & $\begin{array}{l}\text { TLR2, TLR6, and } \\
\text { Dectin-1 }\end{array}$ & No effect \\
\hline Alum & $200 \mu \mathrm{L}$ & Inflammasome & No effect \\
\hline Gardiguimod & $77 \mu \mathrm{g}$ & TLR7 & No effect \\
\hline Poly I:C & $300 \mu \mathrm{g}$ & TLR3 & No effect \\
\hline
\end{tabular}

CAIA, collagen antibody-induced arthritis; LM, lipomannan; LPS, lipopolysaccharide; MPL, monophosphoryl lipid A.

(IgG2a) bind C1' epitope of Cll (359-363; ARGLT), CIIC2 (IgG2b) bind D3 epitope of Cll (687-698; RGAQGPPGATGF), and UL1 (IgG2b) bind U1 epitope of CII (494504; GLVGPRGERGF). Antibodies were purified using $\gamma$-Bind Plus affinity gel matrix (GE Healthcare Bio-Sciences, Uppsala, Sweden). The IgG content was determined from the weight of freeze-dried preparations. The antibody solutions were sterile filtered and stored at $-80^{\circ} \mathrm{C}$ until used.

Male mice over 8 weeks of age were used in all CAIA experiments. CAIA was induced by a single intravenous injection of the four-monoclonal antibody cocktail as described previously. ${ }^{2}$ Arthritis was boosted i.p. 5 or 7 days later with immunostimulants as indicated in Table 1. LPS (from Escherichia coli 055:B5), peptidoglycan (from Staphylococcus aureus), pertussis toxin (from Bordetella pertussis), zymosan A (from Saccharomyce cerevisiae), and aluminum hydroxide gel (alum) were purchased from Sigma-Aldrich, St. Louis, MO. Synthetic monophosphoryl lipid A (MPL), flagellin (from Bacillus subtilis), gardiquimod, poly(I:C), and LM (from Myocobacterium smegmatis) were purchased from InvivoGen, San Diego, CA.

To analyze the first phase of antibody-induced arthritis, all mice subjected to CAIA were pooled and split into age groups of below and above 100 days. Ncf1 genotype differences were statistically analyzed in each group.

\section{Collagen-Induces Arthritis}

CIA was induced under isoflurane anesthesia by injecting 100 $\mu \mathrm{g}$ of rat $\mathrm{Cll}$ (purified from rat chondrosarcoma as described previously) ${ }^{32}$ emulsified in complete Freund's adjuvant (Difco, Detroit, MI) subcutaneously at the base of the tail.

\section{Arthritis Scoring}

Arthritis development in both CAIA and CIA was monitored using a scoring system based on the number of inflamed joints in each paw, inflammation being defined by swelling and redness. ${ }^{33}$ Briefly, one point was given for each swollen and red toe or knuckle and five points for a swollen and red ankle. Maximum score per paw was 15 points, summing up to a maximum 60 points per mouse. 
A

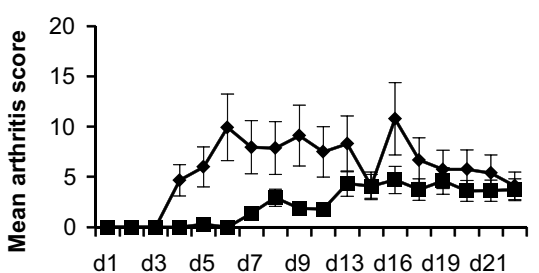

Day after ab cocktail injection

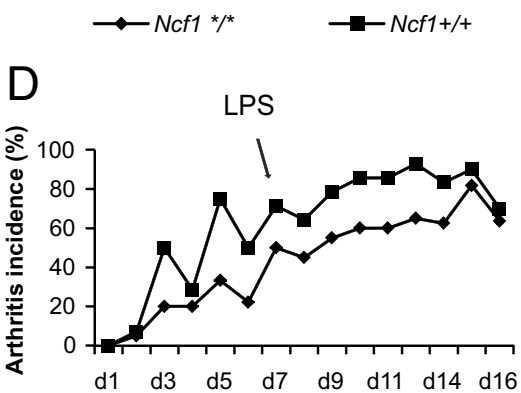

Day after ab cocktail injection

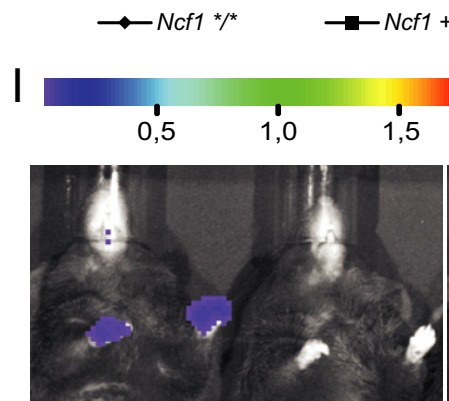

CAIA: $N c f 1+/+\quad$ CAIA: $N c f 1^{* *}$
B

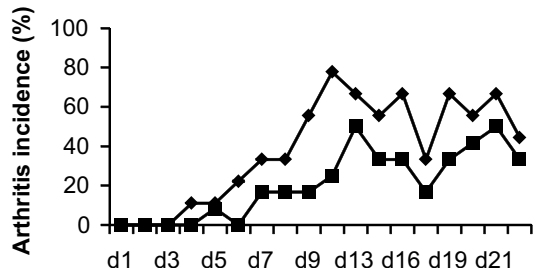

Day after ab cocktail injection

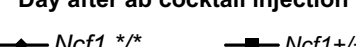

C

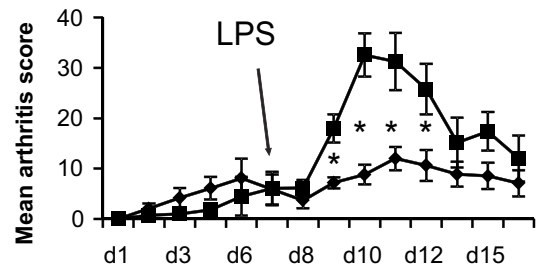

Day after ab cocktail injection

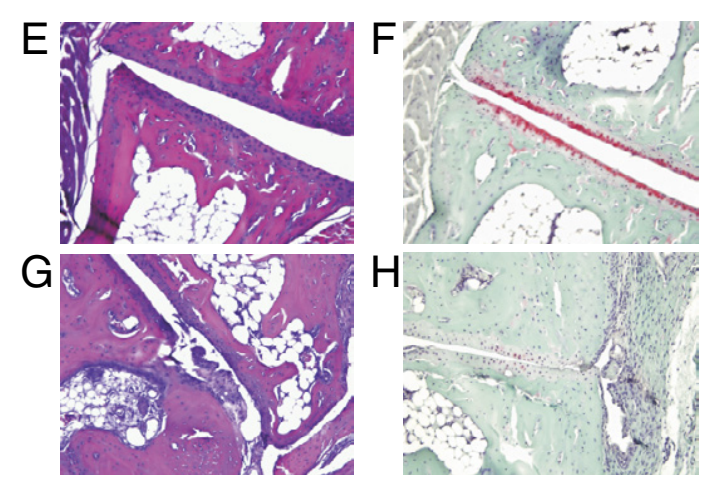

Figure 1. Phagocyte reactive oxygen species (ROS) enhance lipopolysaccharide (LPS) stimulated collagen antibody-induced arthritis (CAIA). CAIA was induced with a single injection of four monoclonal anti-CII antibodies (ab) without additional immune stimulation, and arthritis severity (A) and incidence (B) were followed daily during 3 weeks. $N c f 1$ mutated mice had a tendency to develop more severe arthritis. $N c f 1^{* / *}(n=9), N c f 1^{+/+}$ $(n=12)$. CAIA was stimulated with LPS 7 days after initial antibody cocktail injection in $N c f 1^{* / *}$ and $N c f 1^{+/+}$mice, and arthritis severity (C) and incidence (D) were followed for several days as indicated in the figure. Wild-type B10.Q mice developed more severe CAIA after LPS injection than $N c f 1^{* / *}$ mice. $N c f 1^{* / *}(n=20), N c f 1^{+/+}(n=14)$. Representative HE and safranin staining of joints $(\times 100)$ collected from $N c f 1^{* / *}$ (E and $\mathbf{F}$, respectively) and $\mathrm{Ncfl}^{+/+}$(G and $\mathbf{H}$, respectively) mice during CAIA. In vivo imaging of ROS with L-012 (I) was used to illustrate the severe local inflammation in wild-type mice during LPS stimulated CAIA. ${ }^{*} P<0.05,{ }^{* * *} P<0.01$, and ${ }^{* * * * *} P<0.001$. Mean values and error bars representing SEM are presented; and $n$, given in legend, indicates the number of mice in each group.

\section{L-012 Imaging of ROS Production in Vivo}

Isoflurane-anesthetized mice were injected intraperitoneally with $20 \mathrm{mg} / \mathrm{kg} \mathrm{L}-012$ probe (Wako Chemicals, Neuss, Germany) dissolved in physiological saline. ${ }^{34}$ The luminescent signal was detected with the IVIS 50 bioluminescent system (Xenogen, Alameda, CA), which consists of an anesthesia unit built into a light-tight chamber equipped with a CCD camera 10 minutes after the probe injection. Image acquisition and analysis were performed with Living Image software version 2.50 (Xenogen).

\section{Histology}

Formalin-fixed joints were decalcified in EDTA, Tris, and polyvinylpyrrolidone-containing buffer ( $\mathrm{pH} 6.9)$, dehydrated, and then embedded in paraffin blocks; $5-\mu \mathrm{m}$ sections were stained with either hematoxylin-eosin (HE) or safranin.

\section{IL-6 ELISA}

Serum levels of IL-6 were tested using Mouse IL-6 ELISA Ready-SET-Go! kit (eBioscience, San Diego, CA). Results are reported as fold change from naive mice of respective sex and genotype.

\section{Statistics}

Mann-Whitney U-test was used to perform statistical testing, and arthritis frequencies were analyzed using Fisher's exact test. All $P$ values $<0.05$ were considered as significant $(P<0.05$ is indicated in figures with an asterisk, $P<0.01$ with double asterisks, and $P<0.001$ with triple asterisks). Mean values and error bars representing SEM are shown in all of the figures; and $n$, given in legends, indicates the number of mice in each group.

\section{Results}

\section{LPS Induces Severe Arthritis in the Presence of Functional Phagocyte ROS Production}

On the B10.Q genetic background, antibody injection alone induced only mild arthritis, reaching maximum severity between days 5 and 12 after antibody injection. After a couple of weeks, arthritis subsided, and joints were completely healed in approximately 3 weeks (Figure 1, A and B). In the antibody-induced phase of arthritis, the Ncf1 mutated mice tended to have more severe arthritis than the wild-type animals. A 


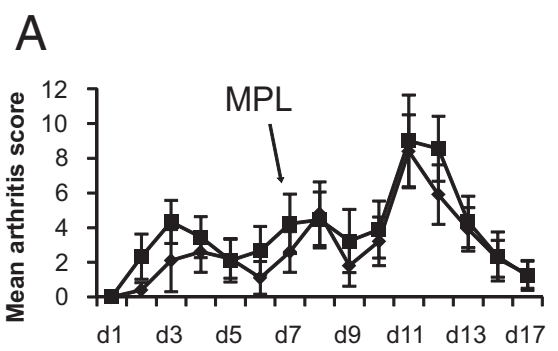

Day after ab cocktail injection

$\rightarrow$ Ncf1*** $\rightarrow$ Ncf1+/+

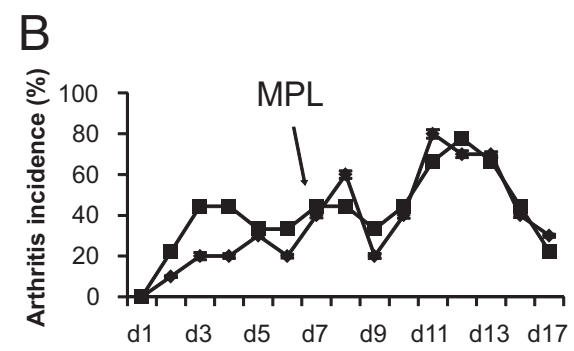

Day after ab cocktail injection
Figure 2. MPL-stimulated collagen antibody-induced arthritis (CAIA). CAIA was stimulated with monophosphoryl lipid A (MPL) 7 days after the antibody (ab) cocktail injection, and arthritis progression was followed until recovery. There were no differences in arthritis severity (A) or incidence (B) between the genotypes. Ncf $1^{* / 4}$ $(n=10), N c f 1^{+/+}(n=9) .{ }^{*} P<0.05,{ }^{* *} P<0.01$, and ${ }^{* *} P<0.001$. Mean values and error bars representing SEM are presented; and $n$, given in legend, indicates the number of mice in each group. strong enhancement of arthritis severity occurs when LPS is injected i.p., and therefore, LPS stimulation is included in the standard protocol for CAIA induction. ${ }^{2}$

Arthritis enhanced with LPS was milder in the Ncf1 mutated mice that lack phagocyte oxidative burst than in the wild-type mice (Figure 1C). The ROS-induced increase in disease severity was not reflected in arthritis incidence (Figure 1D). The genotype difference in arthritis severity at a later stage of the disease is illustrated with representative HE-stained (Figure 1, E and G) and safranin-stained (Figure 1, F and $H$ ) joints. The upper panel (Figure 1, E and F) demonstrates an almost healthy joint collected from an Ncf1 mutated mouse, and the lower panel (Figure 1, G and $\mathrm{H}$ ) shows a highly inflamed joint collected from a wild-type mouse. Severe arthritis in wildtype mice was associated with bone erosion and massive inflammatory polymorphonuclear leukocyte infiltrate in the affected joints. Wild-type synovia also clearly showed a loss of safranin uptake, indicating prominent proteoglycan depletion in the articular cartilage.

In vivo ROS production following neutrophil infiltration in the inflamed paws after LPS injection was illustrated by L-012 imaging (Figure 1I). Arthritis in the Ncf1 wild-type mice was followed by a local massive ROS signal when compared to Ncf1 mutated paws with comparable arthritis scores or paws from healthy wild-type mice.

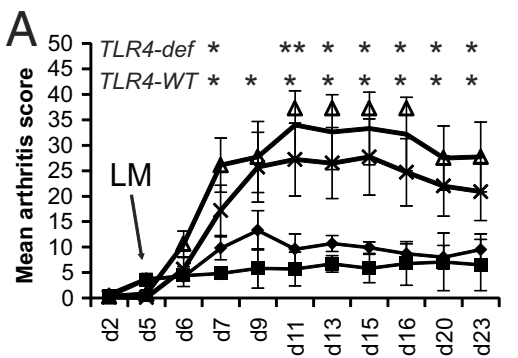

Day after ab cocktail injection

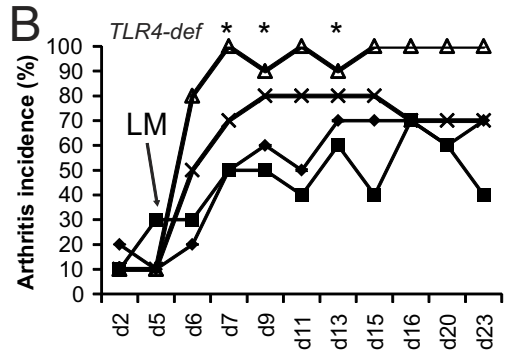

Day after ab cocktail injection sit

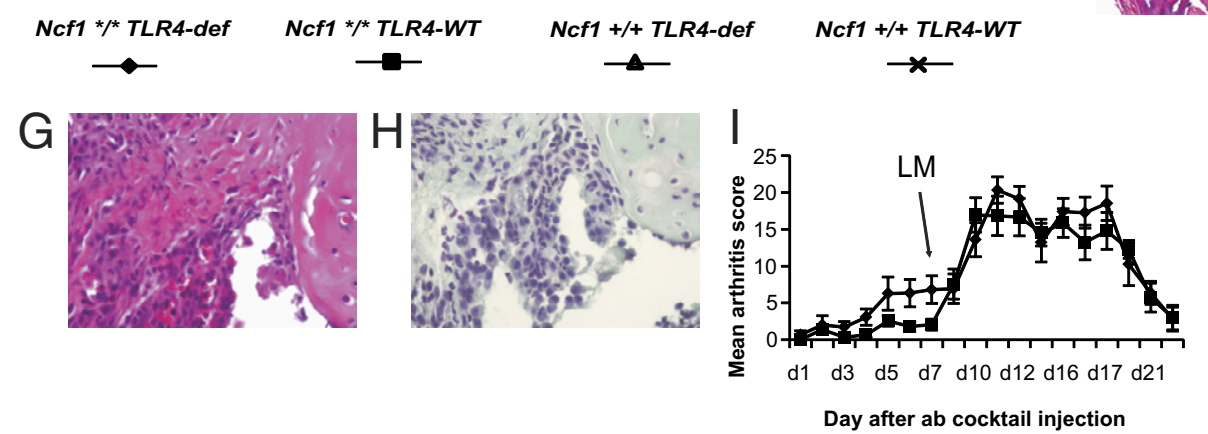

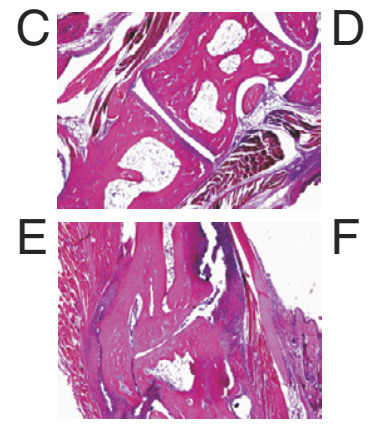

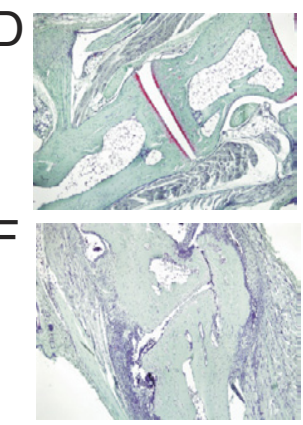

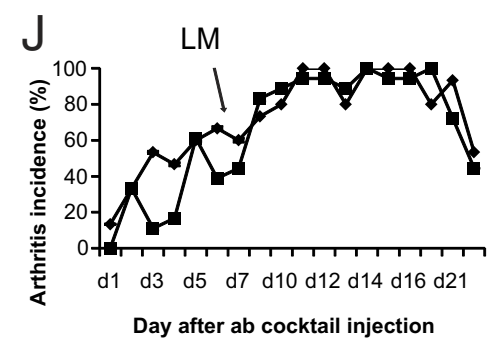

$\longrightarrow M N-$

$\rightarrow-M N+$

Figure 3. Lipomannan (LM)-stimulated collagen antibody-induced arthritis (CAIA). CAIA was induced in $N c f 1^{+/+}$and $N c f 1^{* / *}$ mice with and without functional TLR4 and stimulated with LM 7 days after the antibody transfer. $N c f 1^{+/+}$animals developed more severe arthritis (A) with higher incidence (B) than their wild-type litter mates, whereas the functionality of TLR 4 did not affect arthritis severity or incidence. Asterisks indicate significant differences between $N c f 1^{* / *}$ and $N c f 1^{+/+}$ mice ( $n=10$ in all groups). Representative joints collected from $N c f 1^{* / *}(\mathbf{C}$ and $\mathbf{D})$ and $N c f 1^{+/+}(\mathbf{E}$ and $\mathbf{F})$ mice with LM-stimulated CAIA stained with HE and safranin, respectively $(\times 100)$. Massive neutrophil infiltration was found in the wild-type joint $(\times 400)$ stained with HE (G) and safranin $(\mathbf{H})$, whereas joints from $N c f 1^{* / *}$ mice (C and D) exhibited almost normal joint morphology. CAIA was stimulated 7 days after arthritogenic anti-CII antibody (ab) cocktail injection with LM in transgenic mice with predominant expression of $N c f 1\left(M N^{+}\right)$in macrophages and compared to their completely mutated litter mates ( $M N N^{-}$). There was no difference between the genotypes in arthritis severity (I) or incidence $(\mathbf{J}) . M N(n=15), M N^{+}(n=18)$. ${ }^{*} P<0.05$, ${ }^{* * *} P<0.01$, and ${ }^{* * * *} P<0.001$. Mean values and error bars representing SEM are presented; and $n$, given in legend, indicates the number of mice in each group. 


\section{NOX2 Complex-Derived ROS Enhance LM (TLR2 Agonist) Stimulated CAIA}

LPS is known to stimulate both TLR4 and TLR2 signaling, and to determine which of the LPS-stimulated TLR pathways is regulated by NOX2 complex-derived ROS, CAIA was stimulated with MPL, the arthritogenic, TLR4-specific lipid component of LPS. ${ }^{35}$ MPL induced a mild, but statistically significant enhancement of CAIA, but neither arthritis severity nor incidence was affected by the Ncf1 mutation (Figure 2, A and B), suggesting that TLR4 weakly enhances antibody induced arthritis, while arguing against the role of TLR4 as the receptor mediating ROS-dependent arthritis enhancement.

We continued by testing whether the ROS-dependent increase in CAIA severity was induced by TLR2 stimulation. LM is a TLR agonist that has been reported to signal only via TLR2, and it is known as a TLR4- and TLR6independent stimulant. ${ }^{36}$ Similar to LPS injection, LM injection was followed by significantly more severe arthritis in Ncf1 wild-type mice with intact phagocyte ROS production (Figure 3A). The ROS-induced enhancement of arthritis severity was also accompanied with an increased arthritis incidence (Figure 3B), and the difference in disease severity is illustrated using HE (Figure 3, $C$ and $E$ ) and safranin (Figure 3, D and F) staining of representative joints. Ncf1 mutated mice with milder disease are presented in the upper panel (Figure 3, C and D), and joints collected from wild-type mice with severe arthritis are illustrated in the lower panel (Figure 3, E and F). A clear inflammatory infiltrate accompanied by joint erosion and depletion of proteoglycan was noted in the wildtype joints. Arthritis induced by arthritogenic antibodies and LM is mainly mediated by polymorphonuclear granulocytes as illustrated in the higher magnification $(\times 400)$ images taken from the severely inflamed wild-type joints stained with HE (Figure 3G) and safranin (Figure $3 \mathrm{H}$ ).

When LM-stimulated CAIA was examined in the TLR4deficient mice, TLR4 deficiency do not affect arthritis severity or incidence (Figure 3, A and B). Similar results, supporting the importance of TLR2, but not TLR4, as mediator of ROS-mediated arthritis enhancement, were obtained from both wild-type and $\mathrm{Ncf}^{* / *}$ mice.

\section{Macrophages Do Not Regulate TLR2- Stimulated CAIA}

ROS-dependent suppression of CIA is strongly mediated by macrophages because transgenic mice expressing functional Ncf1 on macrophages are significantly protected against severe arthritis. ${ }^{25}$ By contrast, arthritis severity and incidence (Figure 3, I and J) were not affected by the macrophage-predominant expression of Ncf1 in the CAIA model. These data confirm that ROS-dependent enhancement of arthritis after LM stimulation was not macrophage mediated, but was induced by granulocytes that are the most potent producers of phagocyte-derived ROS in inflamed tissues.

\section{Other Innate Immunity Stimulants Do Not Enhance Antibody-Initiated Arthritis}

To further develop the antibody-induced arthritis model, we continued by testing different innate stimulants' capacity to enhance arthritis. All of the substances, doses, their main receptor targets, and the effect on CAIA are summarized in Table 1. Peptidoglycan was selected as it has been reported to induce mild arthritis after systemic injection in BALB/C and DBA/1 mice, ${ }^{17}$ but in our experimental setting, it failed to enhance CAIA. Similarly, alum, a NALP3 inflammasome activator, ${ }^{37}$ did not enhance antibody-induced joint inflammation.

Pertussis toxin is widely used as an enhancer of central nervous system inflammation in experimental autoimmune encephalomyelitis (an animal model of multiple sclerosis), and its multiple effects on the immune system are known to induce a massive proinflammatory response. Here, no arthritis-enhancing effect could be detected after injection with a standard, highly immunostimulatory dose of pertussis toxin. Similarly zymosan, which triggers arthritis in ZAP70 mutated SKG mice, ${ }^{18}$ failed to enhance joint inflammation in the CAIA model. We also tested whether gardiquimod (a synthetic TLR7

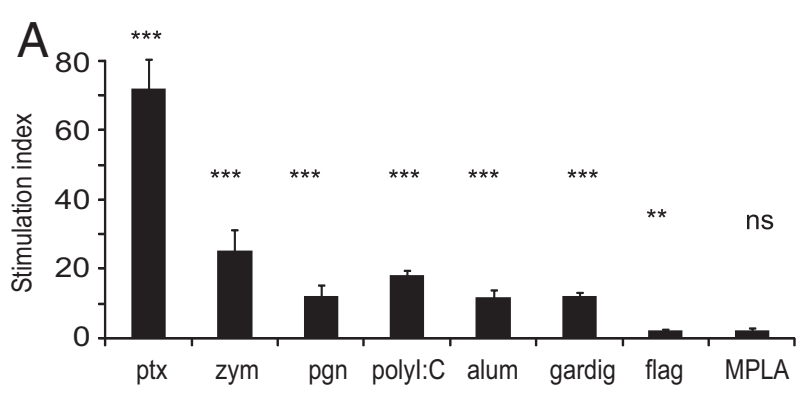

B

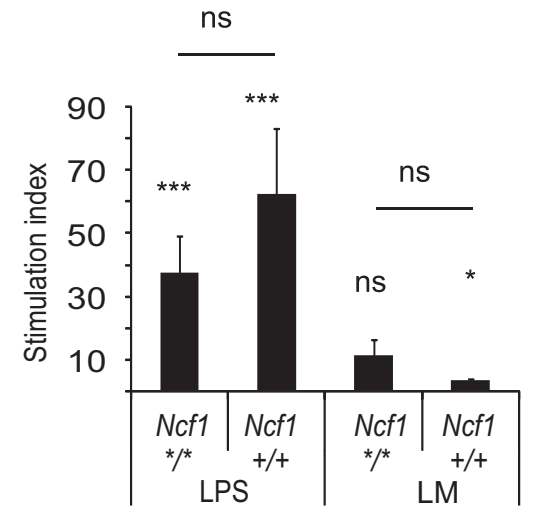

Figure 4. IL-6 responses to immunological stimulants. All stimulants listed in Table 1 were injected into naive mice ( $n=4$ to 8 mice per group) and IL- 6 levels were analyzed from serum samples 24 hours after the i.p. injection. IL-6 levels are reported as stimulation indexes calculated using results from naive mice with the same genotype as background controls. Substances that did not enhance collagen antibody-induced arthritis (CAIA) or enhanced CAIA independently of the animals' reactive oxygen species (ROS) status were only tested in $\mathrm{Ncf1}^{+/+}$mice (A). Substances that stimulated CAIA and whose arthritis-stimulating properties were modulated by ROS were tested in both $N c f 1^{* / * *}$ and $N c f 1^{+/+}$mice $(\mathbf{B}) .{ }^{*} P<0.05,{ }^{* * *} P<0.01$, and ${ }^{* * * *} P<0.001$. Mean values and error bars representing SEM are presented; and $n$, given in legend, indicates the number of mice in each group. flag, flagellin; gardig, gardiquimod; MPL, monophosphoryl lipid A; pgn, peptidoglycan; ptx, pertussis toxin; zym, zymosan. 
agonist) and poly I:C (a synthetic TLR3 agonist) could enhance CAIA, but again, similar to TLR9 stimulation with $\mathrm{CpG}$ oligonucleotides, ${ }^{5}$ no increase in arthritis severity or incidence could be seen.

Lastly, we investigated whether the stimulants tested in the study induced a proinflammatory response in naive mice. Mice were injected at doses described in Table 1, and 24 hours later, serum samples were collected for determination of IL-6 levels. No correlation between the substances' arthritis-enhancing capacity and IL-6 concentration 24 hours after injection in serum was found (Figure 4, A and B). Pertussis toxin, zymosan, peptidoglycan, poly I:C, gardiquimod, and flagellin, all increased serum IL-6 concentration to several fold, even though they did not have any effect on CAIA severity. Conversely, despite their arthritogenicity, MPL and LM induced either a negligible or only modest increase in serum IL-6 levels.

\section{Aged Ncf1 Mutated Mice Develop More Severe Antibody-Induced Arthritis}

The antibody phase of CAIA is likely to be different from the LPS/LM-induced phase. From Figure $1 \mathrm{~A}$, it is evident that there is a trend that the Ncf1 mutated mice developed more severe CAIA than the wild-type mice. To determine whether this is the case, we increased the power of the analysis by taking into account data from all CAIA experiments reported in Figures $1, C$ and D, 2, A and B, $3, I$ and $\mathrm{J}$, and in Table 1. All these experiments were performed in the same animal department to ensure the similarity of the microbiological status of all of the pooled mice, and these mice were split into groups of young
$(<100$ days) and more aged ( $>100$ days old) mice for statistical analysis.

Ncf1 mutation resulting in lowered phagocyte ROS production did not affect the pre-boost phase of CAIA in the younger mice (Figure 5, A and B), but older mice with mutated Ncf1, and thus lacking phagocyte ROS production, were found to develop significantly more severe arthritis than the wild-type controls (Figure 5, C and D).

The presence of ROS-producing neutrophils in the arthritic paws during the first phase of CAIA was confirmed by using L-012 imaging of ROS production. Arthritic paws of the Ncf1 wild-type mice produced a significantly higher signal than the non-arthritic paws from antibody-injected mice or paws from the naive mice (Figure 5E). Neither arthritis nor antibody injection induced any L-012 signal in Ncf1 mutated control mice even in the presence of observable inflammation, confirming the role of NOX2 complex as the main ROS producer in antibodyinduced joint inflammation. This experiment also confirmed that the visual scoring system correlated well with the actual inflammatory infiltrate present in the inflamed joint.

\section{TLR4 Deficiency Does Not Affect CIA Development}

Next, we studied the role of TLR4 in CIA, the most commonly used murine arthritis model, ${ }^{1}$ and we found that neither arthritis severity nor incidence was affected by TLR4 deficiency (Figure 6, A and B). Ncf1*** mice developed more severe arthritis than the wild-type mice, but as in the CAIA model, TLR4 deficiency did not affect arthritis
A

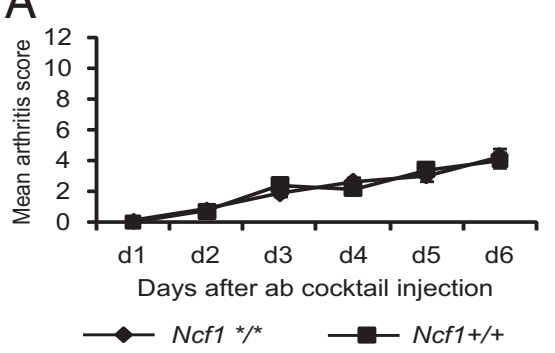

D

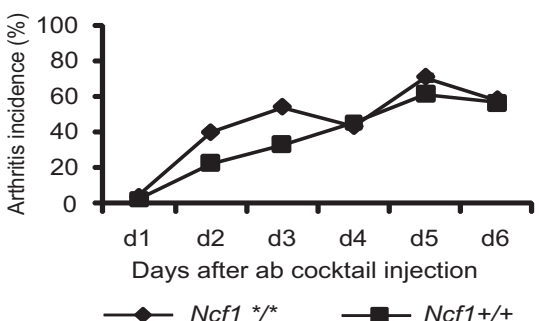

$\mathrm{B}$

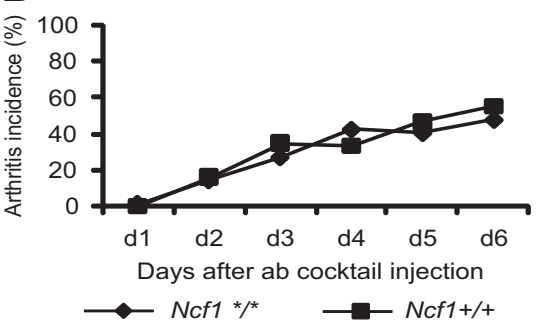

$E$

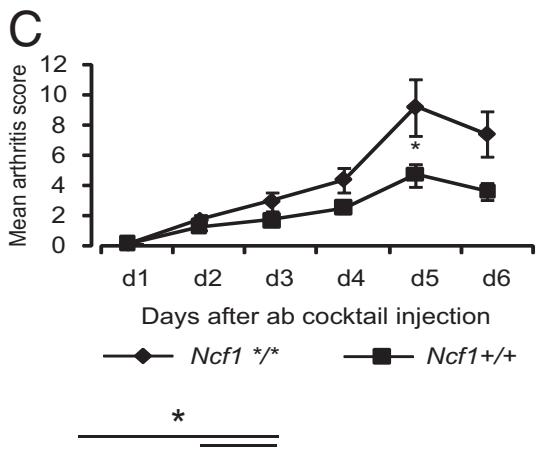

Figure 5. The initial phase of antibody-induced arthritis. In young ( $<100$ days old) mice, Ncf1 mutation did not affect collagen antibody-induced arthritis (CAIA) severity (A) or incidence (B), whereas more aged ( $>100$ days old) $N c f 1$ mutated mice developed more severe $(\mathbf{C})$ arthritis without an effect on arthritis incidence (D) than did the wild-type mice. In young mice, $N c f 1^{* / *} n=82, N c f 1^{+/+} n=69$. In old mice, $N c f 1^{* / *} n=24, N c f 1^{+/+} n=39$. L-012 in vivo imaging of ROS was used to localize ROS production in the inflamed paws with normal ROS production $(\mathbf{E}) . N c f 1^{+/+}$paws emitted significantly increased luminescent signal on arthritis development (Arthritis), whereas antibody-injected paws without clinical signs of arthritis (Ab) or naive paws ( - ) did not give higher signals than paws from ROS-deficient $N c f 1^{* * *}$ mice. ${ }^{*} P<0.05$. Mean values and error bars representing SEM are presented; and $n$, given in legend, indicates the number of mice in each group. RLU, relative light units. 

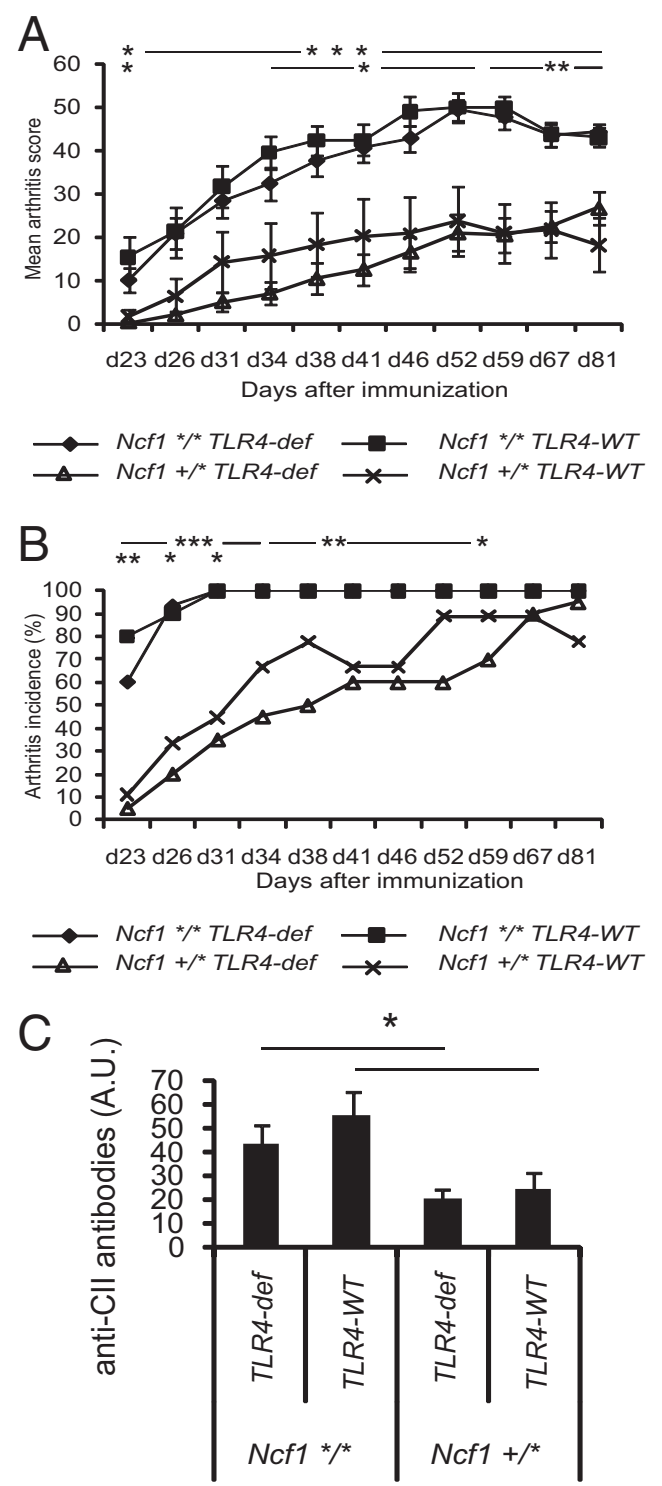

Figure 6. The role of TLR 4 in CIA. CIA was induced with complete Freund's adjuvant and CII in $N c f 1^{* / *}$ and $N c f 1^{+/ *}$ mice with and without functional TLR4. $N c f 1^{* / *}$ mice developed more severe (A) CIA with higher incidence (B) than $N c f 1^{+/ *}$ mice, whereas TLR4 deficiency did not affect CIA severity or incidence (A and $\mathbf{B}$ ) in $N c f 1^{* / *}$ or $N c f 1^{+* *}$ mice. Upper asterisks indicate significances between $N c f 1^{* * *}$ and $N c f 1^{+/ *}$ mice without functional TLR4, and lower asterisks indicate differences with the same $N c f 1$ genotypes all with functional wild-type TLR4. Anti-CII antibody titers were analyzed in all genotypes at day 81 (d81), and they were not affected by TLR4 deficiency (C) As shown in the figure, the enhanced arthritis in $N c f 1^{*}$ mice was followed by a higher level of anti-CII antibody production after CII immunization. ${ }^{*} P<0.05,{ }^{* *} P<0.01$, and ${ }^{* * * *} P<0.001$. Mean values and error bars representing SEM are presented.

phenotype in either wild-type or $\mathrm{Ncf1}^{* / *}$ mice. As published previously, ROS-mediated suppression of CIA in Ncf1 wild-type mice was reflected in lower anti-type-II collagen antibody levels, ${ }^{22}$ whereas the functionality of TLR4 did not affect the antibody response (Figure 6C).

\section{Discussion}

CAIA is a commonly used arthritis model and is developed in two phases; the first phase is induced by the arthritogenic antibodies, and in the second phase, arthritis severity is enhanced by an injection of some immunostimulatory agent. We show here that these two phases are differentially dependent on ROS and rely on different pathogenic mechanisms. We also identified that the ROS-dependent enhancement of arthritis is dependent on stimulation of TLR2 rather than TLR4.

To better define the second, LPS-induced inflammatory phase in antibody-induced arthritis, we addressed the downstream mechanisms that are responsible for arthritis enhancement. LPS stimulates both TLR2 and TLR4, and in light of our experimental evidence, we concluded that the arthritis-amplifying effects are mainly dependent on TLR2, but not TLR4. In fact, LM was highly arthritogenic even in TLR4-deficient mice.

MPL, the lipid component of LPS, is considered as a TLR4-specific stimulant. Here, it induced a mild, but significant, arthritis enhancement, but remarkably, its arthritis-inducing capacity was not dependent on the presence of NOX2 complex-derived ROS, as both Ncf1 mutated and wild-type mice developed arthritis with comparable severity. Thus, we conclude that the ROS-dependent arthritis enhancement is independent of TLR4, whereas TLR4 may mildly stimulate collagen antibodyinduced arthritis via pathways that are not regulated by NOX2 complex-derived ROS. Alternatively, MPL may operate through other innate, arthritis-enhancing pathways.

To further develop the antibody-induced arthritis model, we also addressed whether any other innate stimulant could be more efficient in amplifying arthritis. Surprisingly, out of the array of all tested immunostimulants, only LM and LPS efficiently amplified arthritis, thus leaving TLR2 as the most important TLR receptor for antibody-induced arthritis amplification.

The role of TLR2 in amplification of acute arthritis is consistent with findings from studies using streptococcal cell wall-induced arthritis, ${ }^{38}$ as well as studies with the spontaneous arthritis developing in IL1R-deficient mice. ${ }^{7}$ Additionally, TLR2 blockage has been shown to prevent cytokine release from synovial explant cultures from RA patients, ${ }^{8}$ and TLR2 has also been suggested to induce angiogenesis in inflamed RA synovial tissue. ${ }^{39}$ Recently, TLR2 was also observed to play a role in the pristaneinduced arthritis in rats. ${ }^{40}$

LM is a proinflammatory component of the mycobacterial cell wall ${ }^{41}$ and is considered as a highly specific TLR2 agonist. ${ }^{36,42}$ TLR2 heterodimerizes with TLR1 or TLR6 to induce proinflammatory signaling, ${ }^{43}$ and LM has been shown to induce the formation of TLR2/TLR1 heterodimers, ${ }^{44}$ thus initiating immune responses independently of TLR6. ${ }^{36}$ In LM-stimulated CAIA, the maximum arthritis-enhancing effect was observed in wild-type animals with functional phagocyte oxidative burst. Similarly, inflammation induced by oxidized phospholipids occurring in tissues exposed to large amounts of oxidants was recently reported to be mediated by TLR2. ${ }^{45}$ TLR2 blockage in synovial samples from RA patients has been shown to prevent the release of proinflammatory cytokines. ${ }^{8}$ TLR2 has also been suggested to induce ROS production via direct physical contact between TLR2 and NOX $2,{ }^{46}$ creating a direct link between phagocyte 
ROS production and TLR2. Similarly, TLR2 blocking antibody has been shown to impair ROS production in human eosinophils, further supporting the interplay of NOX2 complex, ROS, and TLR2 as co-inducers of inflammation. ${ }^{42}$

TLR4 has been suggested to play a role in CIA and in some other arthritis models as well as in RA. ${ }^{12,13,47,48}$ To further investigate this, we induced CIA in TLR4-deficient mice. As expected, intact ROS production in the Ncf1 wild-type mice significantly suppressed arthritis development, but surprisingly, genetically encoded TLR4 deficiency affected neither severity nor incidence of CIA. Similarly, TLR4 does not affect the disease course of experimental autoimmune encephalomyelitis, the model of multiple sclerosis, whereas deletion of TLR2 was reported to significantly down-regulate disease severity. ${ }^{49}$

ROS have a complex role in immunity and inflammation. In T cell-driven arthritis models, ROS have been repeatedly shown to suppress inflammation by us and others, ${ }^{21-23,50}$ and at least part of this suppression is mediated by macrophages, because macrophages expressing all functional subunits of the NOX2 complex can reverse the highly arthritogenic Ncf1 mutated genotype..$^{25}$ The ROS-mediated arthritis suppression is regulated by altered T-cell membrane oxidation status, ${ }^{51}$ suggesting that during antigen presentation, macrophages can down-regulate adaptive immune responses by oxidizing the cell membranes of arthritogenic T cells.

An important issue was to investigate the role of ROS in an arthritis model that is not T-cell driven. It should be noted that even if CAIA is not T-cell driven, T cells seem to play a regulatory role in the progression of antibodyinduced inflammation. ${ }^{4,52,53}$ In contrast to the previous findings in the T cell-driven arthritis models, LPS- and LM-stimulated CAIA models were more severe in the wild-type mice with physiological, intact ROS production than in the Ncf1 mutated mice without functional phagocyte ROS production. It has been reported previously that LPS-stimulated CAIA is neutrophil dependent ${ }^{54}$ in similarity with other antibody-induced ${ }^{55}$ and serum-induced arthritis models. ${ }^{56}$ It is likely that the observed ROSdriven enhancement of arthritis was dependent on enhanced neutrophil function because macrophage-specific ROS production was not enough to enhance arthritis, and histological staining revealed a massive neutrophil infiltration in the inflamed joints. Thus, we conclude that granulocytes increase CAIA severity in wild-type mice by producing massive amounts of ROS.

The antibody-induced primary phase of CAIA was, however, more severe in mice lacking ROS, clearly indicating that this phase is dependent on other mechanisms than the following severe arthritis evoked by TLR2 stimulation. Importantly, the effect is not T-cell dependent, as has been shown to be the case in CIA, ${ }^{57}$ but could be more related to the enhanced induction of peritonitis and arthritis by thioglycollate injections in ROS-deficient mice. ${ }^{58,59}$

CAIA is an innate, immunity-driven, T- and B cellindependent arthritis model and is regulated by NOX2 complex-derived ROS. We conclude that the first phase of the antibody-induced disease is suppressed by phagocyte oxidative burst, whereas LM- (TLR2) and LPSstimulated CAIA models are enhanced by NOX2-derived ROS. These findings are important to understand the diverging findings about ROS in autoimmunity and inflammation. ROS have different effects on the inflammatory process, depending on timing and localization of the ROS response.

\section{Acknowledgments}

We thank Emma Mondoc for histology and Tiina Kyrölä, Toni Ilo, and Carlos Palestro for expert care of the experimental animals.

\section{References}

1. Kollias G, Papadaki P, Apparailly F, Vervoordeldonk MJ, Holmdahl R, Baumans V, Desaintes C, Di Santo J, Distler J, Garside P, Hegen M, Huizinga TW, Jungel A, Klareskog L, Mclnnes I, Ragoussis I, Schett G, Hart BT, Tak PP, Toes R, van den Berg W, Wurst W, Gay S: Animal models for arthritis: innovative tools for prevention and treatment. Ann Rheum Dis 2011, 70:1357-1362

2. Nandakumar KS, Holmdahl R: Efficient promotion of collagen antibody induced arthritis (CAIA) using four monoclonal antibodies specific for the major epitopes recognized in both collagen induced arthritis and rheumatoid arthritis. J Immunol Methods 2005, 304:126-136

3. Monach PA, Mathis D, Benoist C: The K/BxN arthritis model. Curr Protoc Immunol 2008, 81:15.22.1-15.22.12

4. Nandakumar KS, Backlund J, Vestberg M, Holmdahl R: Collagen type II (CII)-specific antibodies induce arthritis in the absence of $\mathrm{T}$ or $\mathrm{B}$ cells but the arthritis progression is enhanced by Cll-reactive T cells. Arthritis Res Ther 2004, 6:R544-R550

5. Hultqvist M, Nandakumar KS, Bjorklund U, Holmdahl R: Rabeximod reduces arthritis severity in mice by decreasing activation of inflammatory cells. Ann Rheum Dis 2010, 69:1527-1532

6. Cook AD, De Nardo CM, Braine EL, Turner AL, Vlahos R, Way KJ, Beckman SK, Lenzo JC, Hamilton JA: Urokinase-type plasminogen activator and arthritis progression: role in systemic disease with immune complex involvement. Arthritis Res Ther 2010, 12:R37

7. Abdollahi-Roodsaz S, Joosten LA, Koenders MI, Devesa I, Roelofs MF, Radstake TR, Heuvelmans-Jacobs M, Akira S, Nicklin MJ, Ribeiro-Dias F, van den Berg WB: Stimulation of TLR2 and TLR4 differentially skews the balance of $T$ cells in a mouse model of arthritis. $\mathrm{J}$ Clin Invest 2008, 118:205-216

8. Nic An Ultaigh S, Saber TP, McCormick J, Connolly M, Dellacasagrande J, Keogh B, McCormack W, Reilly M, O'Neill LA, McGuirk P, Fearon U, Veale DJ: Blockade of Toll-like receptor 2 prevents spontaneous cytokine release from rheumatoid arthritis ex vivo synovial explant cultures. Arthritis Res Ther 2011, 13:R33

9. Meng L, Zhu W, Jiang C, He X, Hou W, Zheng F, Holmdahl R, Lu S: Toll-like receptor 3 upregulation in macrophages participates in the initiation and maintenance of pristane-induced arthritis in rats. Arthritis Res Ther 2010, 12:R103

10. Yarilina A, DiCarlo E, Ivashkiv LB: Suppression of the effector phase of inflammatory arthritis by double-stranded RNA is mediated by type I IFNs. J Immunol 2007, 178:2204-2211

11. Ospelt C, Brentano F, Rengel Y, Stanczyk J, Kolling C, Tak PP, Gay RE, Gay S, Kyburz D: Overexpression of toll-like receptors 3 and 4 in synovial tissue from patients with early rheumatoid arthritis: toll-like receptor expression in early and longstanding arthritis. Arthritis Rheum 2008, 58:3684-3692

12. Jung YO, Cho ML, Lee SY, Oh HJ, Park JS, Park MK, Park MJ, Ju JH, Kim SI, Park SH, Kim HY, Min JK: Synergism of toll-like receptor 2 (TLR2). TLR4, and TLR6 ligation on the production of tumor necrosis factor (TNF)-alpha in a spontaneous arthritis animal model of interleukin (IL)-1 receptor antagonist-deficient mice. Immunol Lett 2009, 123:138-143 
13. Roelofs MF, Wenink MH, Brentano F, Abdollahi-Roodsaz S, OppersWalgreen B, Barrera P, van Riel PL, Joosten LA, Kyburz D, van den Berg WB, Radstake TR: Type I interferons might form the link between Toll-like receptor (TLR) 3/7 and TLR4-mediated synovial inflammation in rheumatoid arthritis (RA). Ann Rheum Dis 2009, 68:1486-1493

14. Hayashi T, Gray CS, Chan M, Tawatao RI, Ronacher L, McGargill MA, Datta SK, Carson DA, Corr M: Prevention of autoimmune disease by induction of tolerance to Toll-like receptor 7. Proc Natl Acad Sci U S A 2009, 106:2764-2769

15. Sacre SM, Lo A, Gregory B, Simmonds RE, Williams L, Feldmann M, Brennan FM, Foxwell BM: Inhibitors of TLR8 reduce TNF production from human rheumatoid synovial membrane cultures. J Immunol 2008, 181:8002-8009

16. Asagiri M, Hirai T, Kunigami T, Kamano S, Gober HJ, Okamoto K, Nishikawa K, Latz E, Golenbock DT, Aoki K, Ohya K, Imai Y, Morishita Y, Miyazono K, Kato S, Saftig P, Takayanagi H: Cathepsin K-dependent toll-like receptor 9 signaling revealed in experimental arthritis. Science 2008, 319:624-627

17. Koga T, Kakimoto K, Hirofuji T, Kotani S, Ohkuni H, Watanabe K, Okada N, Okada H, Sumiyoshi A, Saisho K: Acute joint inflammation in mice after systemic injection of the cell wall, its peptidoglycan, and chemically defined peptidoglycan subunits from various bacteria. Infect Immun 1985, 50:27-34

18. Yoshitomi H, Sakaguchi N, Kobayashi K, Brown GD, Tagami T, Sakihama T, Hirota K, Tanaka S, Nomura T, Miki I, Gordon S, Akira S, Nakamura T, Sakaguchi S: A role for fungal \{beta\}-glucans and their receptor Dectin-1 in the induction of autoimmune arthritis in genetically susceptible mice. J Exp Med 2005, 201:949-960

19. Rosenzweig HL, Clowers JS, Nunez G, Rosenbaum JT, Davey MP Dectin-1 and NOD2 mediate cathepsin activation in zymosan-induced arthritis in mice. Inflamm Res 2011, 60:705-714

20. Lee EK, Kang SM, Paik DJ, Kim JM, Youn J: Essential roles of Toll-like receptor-4 signaling in arthritis induced by type II collagen antibody and LPS. Int Immunol 2005, 17:325-333

21. Olofsson P, Holmberg J, Tordsson J, Lu S, Akerstrom B, Holmdahl R: Positional identification of $\mathrm{Ncf1}$ as a gene that regulates arthritis severity in rats. Nat Genet 2003, 33:25-32

22. Hultqvist M, Olofsson P, Holmberg J, Backstrom BT, Tordsson J, Holmdahl R: Enhanced autoimmunity, arthritis, and encephalomyelitis in mice with a reduced oxidative burst due to a mutation in the Ncf1 gene. Proc Natl Acad Sci U S A 2004, 101:12646-12651

23. Hagenow K, Gelderman KA, Hultqvist M, Merky P, Backlund J, Frey O, Kamradt T, Holmdahl R: Ncf1-associated reduced oxidative burst promotes IL-33R+ T cell-mediated adjuvant-free arthritis in mice. J Immunol 2009, 183:874-881

24. Holmdahl R, Klareskog L, Andersson M, Hansen C: High antibody response to autologous type II collagen is restricted to $\mathrm{H}-2$ q. Immunogenetics 1986, 24:84-89

25. Gelderman KA, Hultqvist M, Pizzolla A, Zhao M, Nandakumar KS, Mattsson R, Holmdahl R: Macrophages suppress T cell responses and arthritis development in mice by producing reactive oxygen species. J Clin Invest 2007, 117:3020-3028

26. Coutinho A, Meo T: Genetic basis for unresponsiveness to lipopolysaccharide in C57BL/10Cr mice. Immunogenetics 1978, 7:17-24

27. Poltorak A, He X, Smirnova I, Liu MY, Van Huffel C, Du X, Birdwell D, Alejos E, Silva M, Galanos C, Freudenberg M, Ricciardi-Castagnoli P, Layton B, Beutler B: Defective LPS signaling in C3H/HeJ and C57BL/ 10ScCr mice: mutations in TIr4 gene. Science 1998, 282:2085-2088

28. Thomas JA, Tsen MF, White DJ, Horton JW: TLR4 inactivation and rBPI(21) block burn-induced myocardial contractile dysfunction. Am J Physiol Heart Circ Physiol 2002, 283:H1645-H1655

29. Holmdahl R, Rubin K, Klareskog L, Larsson E, Wigzell H: Characterization of the antibody response in mice with type II collagen-induced arthritis, using monoclonal anti-type II collagen antibodies. Arthritis Rheum 1986, 29:400-410

30. Schulte S, Unger C, Mo JA, Wendler O, Bauer E, Frischholz S, von der Mark K, Kalden JR, Holmdahl R, Burkhardt H: Arthritis-related B cell epitopes in collagen II are conformation-dependent and sterically privileged in accessible sites of cartilage collagen fibrils. J Biol Chem 1998, 273:1551-1561

31. Bajtner E, Nandakumar KS, Engstrom A, Holmdahl R: Chronic development of collagen-induced arthritis is associated with arthritogenic antibodies against specific epitopes on type II collagen. Arthritis Res Ther 2005, 7:R1148-R1157
32. Smith BD, Martin GR, Miller EJ, Dorfman A, Swarm R: Nature of the collagen synthesized by a transplanted chondrosarcoma. Arch Biochem Biophys 1975, 166:181-186

33. Holmdahl R, Carlsen S, Mikulowska A, Vestberg M, Brunsberg U, Hansson AS, Sundvall M, Jansson L, Pettersson U: Genetic analysis of mouse models for rheumatoid arthritis. Human Genome Methods. Edited by Adolph KW. New York, CRC, 1998, pp. 215-238

34. Kielland A, Blom T, Nandakumar KS, Holmdahl R, Blomhoff R, Carlsen $\mathrm{H}$ : In vivo imaging of reactive oxygen and nitrogen species in inflammation using the luminescent probe L-012. Free Radic Biol Med 2009, 47:760-766

35. Yoshino S, Ohsawa M: The role of lipopolysaccharide injected systemically in the reactivation of collagen-induced arthritis in mice. $\mathrm{Br} J$ Pharmacol 2000, 129:1309-1314

36. Quesniaux VJ, Nicolle DM, Torres D, Kremer L, Guerardel Y, Nigou J, Puzo G, Erard F, Ryffel B: Toll-like receptor 2 (TLR2)-dependentpositive and TLR2-independent-negative regulation of proinflammatory cytokines by mycobacterial lipomannans. J Immunol 2004, 172 : 4425-4434

37. Eisenbarth SC, Colegio OR, O'Connor W, Sutterwala FS, Flavell RA: Crucial role for the Nalp3 inflammasome in the immunostimulatory properties of aluminium adjuvants. Nature 2008, 453:1122-1126

38. Abdollahi-Roodsaz S, Joosten LA, Helsen MM, Walgreen B, van Lent $\mathrm{PL}$, van den Bersselaar LA, Koenders MI, van den Berg WB: Shift from toll-like receptor 2 (TLR-2) toward TLR-4 dependency in the erosive stage of chronic streptococcal cell wall arthritis coincident with TLR-4-mediated interleukin-17 production. Arthritis Rheum 2008 58:3753-3764

39. Saber T, Veale DJ, Balogh E, McCormick J, Nicanultaigh S, Connolly $\mathrm{M}$, Fearon U: Toll-like receptor 2 induced angiogenesis and invasion is mediated through the Tie2 signalling pathway in rheumatoid arthritis. PLoS One 2011, 6:e23540

40. Zhu W, Meng L, Jiang C, Hou W, Xu J, Wang B, Lu S: Induction of toll-like receptor 2 positive antigen-presenting cells in spleen of pristane-induced arthritis in rats. Mol Biol Rep 2011, 39:3667-3673

41. Dao DN, Kremer L, Guerardel Y, Molano A, Jacobs WR Jr., Porcelli SA, Briken V: Mycobacterium tuberculosis lipomannan induces apoptosis and interleukin-12 production in macrophages. Infect Immun 2004, 72:2067-2074

42. Driss V, Legrand F, Hermann E, Loiseau S, Guerardel Y, Kremer L, Adam E, Woerly G, Dombrowicz D, Capron M: TLR2-dependent eosinophil interactions with mycobacteria: role of alpha-defensins. Blood 2009, 113:3235-3244

43. Ozinsky A, Underhill DM, Fontenot JD, Hajjar AM, Smith KD, Wilson CB, Schroeder L, Aderem A: The repertoire for pattern recognition of pathogens by the innate immune system is defined by cooperation between toll-like receptors. Proc Natl Acad Sci U S A 2000, 97: 13766-13771

44. Elass E, Aubry L, Masson M, Denys A, Guerardel Y, Maes E, Legrand D, Mazurier J, Kremer L: Mycobacterial lipomannan induces matrix metalloproteinase- 9 expression in human macrophagic cells through a Toll-like receptor 1 (TLR1)/TLR2- and CD14-dependent mechanism. Infect Immun 2005, 73:7064-7068

45. Kadl A, Sharma PR, Chen W, Agrawal R, Meher AK, Rudraiah S, Grubbs N, Sharma R, Leitinger N: Oxidized phospholipid-induced inflammation is mediated by Toll-like receptor 2. Free Radic Biol Med 2011, 51:1903-1909

46. Yang C, Shin D, Kim K, Lee Z, Lee C, Park SG, Bae YS, Jo E: NADPH oxidase 2 interaction with TLR2 is required for efficient innate immune responses to mycobacteria via cathelicidin expression. J Immunol 2009, 182:3696-3705

47. Abdollahi-Roodsaz S, Joosten LA, Roelofs MF, Radstake TR, Matera G, Popa C, van der Meer JW, Netea MG, van den Berg WB: Inhibition of Toll-like receptor 4 breaks the inflammatory loop in autoimmune destructive arthritis. Arthritis Rheum 2007, 56:2957-2967

48. Abdollahi-Roodsaz S, Joosten LA, Koenders MI, van den Brand BT, van de Loo FA, van den Berg WB: Local interleukin-1-driven joint pathology is dependent on toll-like receptor 4 activation. Am J Pathol 2009, 175:2004-2013

49. Miranda-Hernandez S, Gerlach N, Fletcher JM, Biros E, Mack M, Korner H, Baxter AG: Role for MyD88, TLR2 and TLR9 but not TLR1, TLR4 or TLR6 in experimental autoimmune encephalomyelitis. J Immunol 2011, 187:791-804 
50. George-Chandy A, Nordstrom I, Nygren E, Jonsson IM, Postigo J, Collins LV, Eriksson K: Th17 development and autoimmune arthritis in the absence of reactive oxygen species. Eur J Immunol 2008, 38:1118-1126

51. Gelderman KA, Hultqvist M, Holmberg J, Olofsson P, Holmdahl R: T cell surface redox levels determine $T$ cell reactivity and arthritis susceptibility. Proc Natl Acad Sci U S A 2006, 103:12831-12836

52. Wang J, Fathman JW, Lugo-Villarino G, Scimone L, von Andrian U, Dorfman DM, Glimcher LH: Transcription factor T-bet regulates inflammatory arthritis through its function in dendritic cells. J Clin Invest 2006, 116:414-421

53. Mitamura M, Nakano N, Yonekawa T, Shan L, Kaise T, Kobayashi T, Yamashita K, Kikkawa H, Kinoshita M: T cells are involved in the development of arthritis induced by anti-type II collagen antibody. Int Immunopharmacol 2007, 7:1360-1368

54. Nandakumar KS, Svensson L, Holmdahl R: Collagen type II-specific monoclonal antibody-induced arthritis in mice: description of the disease and the influence of age, sex, and genes. Am J Pathol 2003, 163:1827-1837

55. Tanaka D, Kagari T, Doi H, Shimozato T: Essential role of neutrophils in anti-type II collagen antibody and lipopolysaccharide-induced arthritis. Immunology 2006, 119:195-202
56. Monach PA, Nigrovic PA, Chen M, Hock H, Lee DM, Benoist C, Mathis D: Neutrophils in a mouse model of autoantibody-mediated arthritis: critical producers of Fc receptor gamma, the receptor for C5a, and lymphocyte function-associated antigen 1. Arthritis Rheum 2010, 62:753-764

57. Hultqvist M, Bäcklund J, Bauer K, Gelderman KA, Holmdahl R: Lack of reactive oxygen species breaks T cell tolerance to collagen type II and allows development of arthritis in mice. J Immunol 2007, 179: 1431-1437

58. Jackson SH, Gallin JI, Holland SM: The P47phox mouse knock-out model of chronic granulomatous disease. J Exp Med 1995, 182 : $751-758$

59. van de Loo FA, Bennink MB, Arntz OJ, Smeets RL, Lubberts $E$ Joosten LA, van Lent PL, Coenen-de Roo CJ, Cuzzocrea S, Segal BH, Holland SM, van den Berg WB: Deficiency of NADPH oxidase components p47phox and gp91phox caused granulomatous synovitis and increased connective tissue destruction in experimental arthritis models. Am J Pathol 2003, 163:1525-1537 